\title{
Kinematics of prestack shot-geophone migration
}

\author{
Christiaan C. Stolk*, University of Twente, Maarten V. de Hoop, Colorado School of Mines, and William \\ W. Symes, Rice University
}

\section{Summary}

Prestack migration methods based on data binning produce kinematic artifacts, i.e. coherent events not corresponding to actual reflectors, in the prestack image volume, when the medium is strongly refracting. Shot-geophone ("survey sinking") migration, on the other hand, does not produce such artifacts when events to be migrated arrive in the data along non-turning rays. In contrast to prestack migration methods based on data binning, common image gathers produced by shot-geophone migration exhibit the appropriate semblance property in either offset domain (focussing at zero offset) or angle domain (focussing at zero slope), when the migration velocity is kinematically correct. Thus shot-geophone migration may be a particularly appropriate tool for migration velocity analysis of data exhibiting structural complexity.

\section{Introduction}

The basis of migration velocity analysis is the semblance principle: prestack migrated data volumes contain flat image gathers, i.e. are at least kinematically independent of the bin or stacking parameter, when the velocity is correct (Yilmaz, 1987). However, complex velocity structure generates strong refraction, hence multiple raypaths connecting source and receiver locations with reflection points. Multiple raypaths in turn imply that the semblance principle is not valid: that is, image gathers are not in general flat, even when the migration velocity closely approximates the true propagation velocity (Stolk and Symes, 2004)

The failure of the semblance principle in complex structure afflicts all prestack migration techniques based on data binning, i.e. for which each data bin creates an independent image. This category includes many variants of common shot, common offset and common scattering angle migration - see Nolan and Symes (1996, 1997); Xu et al. (2001); Brandsberg-Dahl et al. (2003); Stolk and Symes (2004).

One well-known form of prestack image formation does not migrate image bins independently: this is Claerbout's (1985) survey-sinking migration, or shot-geophone migration, commonly implemented using some variety of oneway wave equation to extrapolate source and receiver depths, either simultaneously (DSR migration) or separately (shot profile migration). Such depth extrapolation implementation presumes that rays carrying significant energy travel essentially vertically (the "DSR condition", per Stolk and De Hoop (2001)).
This paper demonstrates that a semblance principle appropriate for DSR migration holds regardless of velocity field complexity, even in the presence of multipathing, assuming

- the DSR condition,

- enough data to determine wavefield kinematics (for example, areal or "true 3D" acquisition in general, or narrow azimuth data plus mild cross-line heterogeneity), and

- a kinematically correct migration velocity field.

This result was established by Stolk and De Hoop (2001). We outline here a somewhat simpler derivation of this property, and provide a $2 \mathrm{D}$ synthetic illustration. This semblance principle takes several roughly equivalent forms, corresponding to several available methods for forming image gathers. Schultz and Sherwood (1982) and Claerbout (1985) suggest a definition of image gather depending on (subsurface) offset and depth: in such offset image gathers, energy is focussed at zero offset when the velocity is kinematically correct. De Bruin et al. (1990) and Prucha et al. (1999) suggest one method of forming angle image gathers, while Sava and Fomel (2003) suggest another: such gathers are functions of scattering angle and depth. Common angle gathers, extracted from angle image volumes of either type, each image the subsurface, so should be compatible. That is, correct migration velocity flattens both types of angle image gather.

In a companion paper (Stolk et al., 2005), we show that DSR migration is kinematically equivalent to shot profile migration, and to variants of two-way reverse time and Kirchhoff migration: all of these shot-geophone migration methods satisfy the appropriate semblance principle under the conditions stated above. In particular, depth extrapolation per se is not the source of the good kinematic properties of shot-geophone migration. Note also that angle imaging via shot-geophone migration, is not equivalent, even kinematically, to the surface-oriented common angle imaging described by Xu et al. (2001); BrandsbergDahl et al. (2003) - indeed, the latter typically generates kinematic artifacts when multiple ray paths carry important energy.

For arbitrary 3D complexity in the migration velocity field, validity of the semblance principle requires areal coverage ("true 3D" data). In particular we cannot guarantee the absence of kinematic artifacts in shot-geophone migration of narrow azimuth data, unless more constraints are placed on the velocity model, e.g. mild crossline heterogeneity. 


\section{Kinematics of prestack shot-geophone migration}

In the remainder of this paper we consider first the relations between events in the data and on the one hand the physical reflector, and on the other hand the reflectors in the migrated data. We sketch a ray-theoretic analysis which establishes these relationships. We then present an example using 2D synthetic data illustrating the semblance property. The example contrasts the angle image gathers produced by (Kirchhoff) common scattering angle migration with those produced by shot-geophone migration. Kinematic artifacts appear in the former but not the latter.

\section{Kinematics of data downward continuation}

An event in the data is characterized by its (3D) moveout: locally, by a moveout equation $t=T\left(\mathbf{x}_{s}, \mathbf{x}_{r}\right)$, and infinitesimally by the source and receiver slownesses

$$
\mathbf{p}_{s}=\nabla_{\mathbf{x}_{s}} T, \mathbf{p}_{r}=\nabla_{\mathbf{x}_{r}} T
$$

Significant energy with this moveout implies that locally near $\left(\mathbf{x}_{s}, \mathbf{x}_{r}, t\right)$ the data contains a plane wave component with wavenumber $\left(\omega \mathbf{p}_{s}, \omega \mathbf{p}_{r}, \omega\right)$. These coordinates (position, wavenumber) give the phase space representation of the event.

Assume that the frequencies above are high enough relative to the length scales in the velocity that such local plane wave components propagate according to geometric acoustics. This assumption tacitly underlies much of reflection processing, and in particular is vital to the success of migration.

The first step in the analysis concerns the relationship for single scattering between a data event, say localized around the position $\left(\mathbf{x}_{s}, \mathbf{x}_{r}, t\right)$, and wavevector $\left(\omega \mathbf{p}_{s}, \omega \mathbf{p}_{r}, \omega\right)$, and a reflector say with phase space coordinates $(\mathbf{y}, \mathbf{k})$. The relationship is depicted in Figure 1. The two-vector $\mathbf{p}_{s}$ determines a three vector $\left(p_{s, x}, p_{s, y}, p_{s, z}\right)$ through the constraint $\left\|\left(p_{s, x}, p_{s, y}, p_{s, z}\right)\right\|=\frac{1}{v\left(\mathbf{x}_{s}\right)}$. The shot position $\mathbf{x}_{s}$ together with this three vector determines a ray, the position and slowness coordinates of which we denote by $\mathbf{X}_{s}(t)$ and $\mathbf{P}_{s}(t)$, with $\mathbf{X}_{s}(0)=$ $\mathbf{x}_{s}, \mathbf{P}_{s}(0)=-\left(p_{s, x}, p_{s, y}, p_{s, z}\right)$. This ray hits the scattering point at some time, say $\mathbf{X}\left(t_{s}\right)=\mathbf{y}$. From the scattering point the receiver ray takes off, denoted by $\mathbf{X}_{r}(t)$ and $\mathbf{P}_{r}(t)$, where we let $\mathbf{X}_{r}\left(t_{s}\right)=\mathbf{X}_{s}\left(t_{s}\right)=\mathbf{y}$. This must be the ray determined by $\mathbf{x}_{r}$ and $\mathbf{p}_{r}$. The wavevector $\mathbf{k}$ satisfies

$$
\omega \mathbf{P}_{r}\left(t_{s}\right)=\omega \mathbf{P}\left(t_{s}\right)+\mathbf{k} .
$$

The wavevector $\mathbf{k}$ is normal to the reflector, which leads to the usual kinematics.

How is such an event migrated by a DSR wavefield extrapolation procedure? With the geometrical acoustics assumption, a one-way extrapolator propagates energy along rays. A propagator with initial depth $z$, and final depth $Z$, propagates a local wave front, say at a phase space point $\left(x, y, t, p_{x}, p_{y}\right)$ at depth $z$ along the ray determined by $\mathbf{x}=(x, y, z)$ and $\mathbf{p}=\left(p_{x}, p_{y}\right)$, until the ray hits the $Z$-plane. Here it is assumed that the velocity along

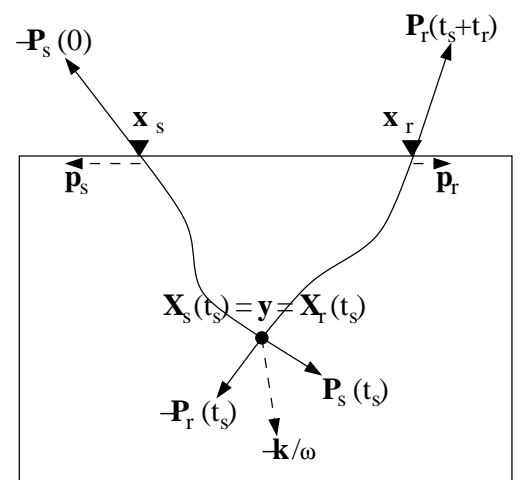

Fig. 1: Ray theoretic relation between data event and physical (single) reflector.

the ray does not become horizontal or too close to horizontal as extrapolators do not, or poorly, propagate such waves.

Let the downward continued data be denoted in midpoint half-offset notation by

$$
D\left(X, Y, Z, H_{x}, H_{y}, T\right) .
$$

Subsurface source/receiver type coordinates are given as usual by $\left(X_{s}, Y_{s}\right)=\left(X-H_{x}, Y-H_{y}\right)$ and $\left(X_{r}, Y_{r}\right)=$ $\left(X+H_{x}, Y+H_{y}\right)$. Double-square-root downward continuation can be viewed as two consecutive one-way extrapolations. First the data is seen as a set of shot records that are downward continued in the receiver coordinate. An event with phase space coordinates $\left(\mathbf{x}_{s}, \mathbf{x}_{r}, t\right)$ and $\left(\omega \mathbf{p}_{s}, \omega \mathbf{p}_{r}, \omega\right)$, the event is migrated along the ray determined by $\left(\mathbf{x}_{r}, \mathbf{p}_{r}\right)$. Next the thus obtained data is seen as a set of receiver records that are downward continued in the source and time coordinates. It follows that with a data event, say at $\left(\mathbf{x}_{s}, \mathbf{x}_{r}, t, \omega \mathbf{p}_{s}, \omega \mathbf{p}_{r}, \omega\right)$, at depth $z$, are associated a ray through the receiver point, determined by $\left(x_{r}, y_{r}, z\right)$ and $\mathbf{p}_{r}$, and a ray through the source point, determined by $\left(x_{s}, y_{s}, z\right)$ and $\mathbf{p}_{s}$. If $\left(X_{s}, Y_{s}, X_{r}, Y_{r}, T, \omega \mathbf{P}_{s}, \omega \mathbf{P}_{r}, \omega\right)$ are the phase space coordinates of the event at depth $Z$, then we have:

- $\left(X_{s}, Y_{s}, Z, \mathbf{P}_{s}\right)$ is on the ray determined by $\left(x_{s}, y_{s}, z, \mathbf{p}_{s}\right)$, say after time $\Delta t_{s}$;

- $\left(X_{r}, Y_{r}, Z, \mathbf{P}_{r}\right)$ is on the ray determined by $\left(x_{r}, y_{r}, z, \mathbf{p}_{r}\right)$, say after time $\Delta t_{r}$

- for the difference in time of the event there is the relation

$$
t-T=\Delta t_{s}+\Delta t_{r} .
$$

For each depth $z$ there is a unique associated event in the downward continued data, which is an essential difference with the Kirchhoff approximation, where there can be multiple migrated events. The time of the downward continued event decreases with the depth. Therefore, there is a unique depth where the time associated with the downward continued event is zero. At $T=0$, the downward 


\section{Kinematics of prestack shot-geophone migration}

continued event is at the physical reflection point at zero offset $\mathbf{X}_{s}=\mathbf{X}_{r}=\mathbf{y}$. This shows the semblance principle for the offset image gathers, since they are obtained by taking the downward continued data $D$ at $t=0$.

The assumption of complete data comes in here in an essential way. For marine data, crossline wavenumber information may be limited. If there are several crossline wavenumbers, with which an observed event is compatible, then migration along several ray pairs can occur. Thus application of the imaging condition in the crossline direction may not select a unique image reflector for each event.

\section{DSR angle gathers}

The DSR angle gathers of De Bruin et al. (1990); Prucha et al. (1999) are obtained from the downward continued data by taking a Radon transform in offset and time, with slant parameter $\mathbf{p}=\left(p_{x}, p_{y}\right)$ :

$B\left(\mathbf{X}, p_{x}, p_{y}\right)=\iint d H_{x} d H_{y} D\left(\mathbf{X}, H_{x}, H_{y}, p_{x} H_{x}+p_{y} H_{y}\right)$.

We view this as an integration over planes in the $\left(H_{x}, H_{y}, T\right)$ space, the planes given by $T=p_{x} H_{x}+p_{y} H_{y}$.

The ray-theoretical arguments above show that at time $T=0$, energy is at offset $\left(H_{x}, H_{y}\right)=0$. For small times, the migrated event are at small offsets, in fact one can show that the offset coordinates satisfy

$$
2 \sqrt{H_{x}^{2}+H_{y}^{2}} \leq|T| v_{\|, \max } .
$$

Here $v_{\|, \max }$ is an upperbound for the length of the horizontal component of ray velocity in some region around the midpoint $\mathbf{x}$. So the locations of the migrated events are in a cone in the $\left(H_{x}, H_{y}, T\right)$ space. For the $2 \mathrm{D}$ case this is displayed as the shaded part in Figure 2.

The parameters $p_{x}, p_{y}$ can be related to the angle in an angle gather. Let us assume there is a maximum value of $\sqrt{p_{x}^{2}+p_{y}^{2}}$ to be used, assume specifically

$$
\sqrt{p_{x}^{2}+p_{y}^{2}}<\frac{2}{v_{\|, \max }}
$$

It then follows that the integration planes $T=p_{x} H_{x}+$ $p_{y} H_{y}$ of (1) are in the white region of Figure 2. Hence they intersect the cones above only at $\left(H_{x}, H_{y}\right)=0$ and $T=0$. It follows that all contributions to the angle gathers from single scattered events (assuming the correct velocity) model, appear at the physical scattering point independent of the angle parameterized by $\left(p_{x}, p_{y}\right)$. I.e., the semblance principle holds.

\section{Examples}

In a $2 \mathrm{D}$ synthetic data example, we illustrate the dramatic contrast between the behaviors of forming image

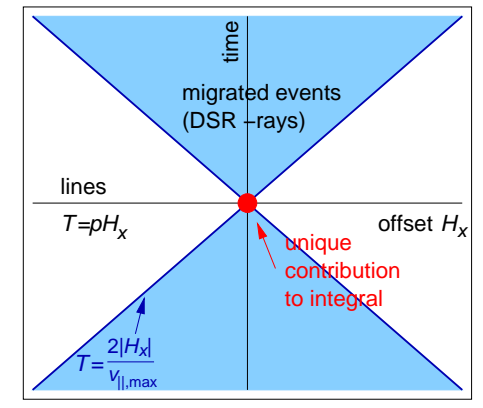

Fig. 2: Region in the offset-time plane where migrated data events are located (shaded), and region contributing to the angle gathers $B$ (white). The only contribution comes therefore from the origin $H_{x}=0, H=0$.

(or common-image-point) gathers by wavefield extrapolation migration and forming image gathers by other forms of prestack depth migration. In the example, the cause of the differences is the formation of caustics, while the DSR assumption is - for the acquisition offsets considered - satisfied. For the wavefield extrapolation migration we employ an approach using the DSR equation and a generalized screen propagator (GSP) implementation Le Rousseau and De Hoop (2001); we form angle image gathers by Radon transform in offset and time. Conversion of 'slope' to scattering angle is carried out based on (De Hoop et al., 2003, (88)-(89)).

Our example is used in Brandsberg-Dahl et al. (2003). The compressional-wave velocity model (Figure 3 (left)) is a simplication of the geological setting of the Valhall field. The model is in fact isotropic elastic, but the main heterogeneity appears in the compressional wave velocity. It consists of a slow Gaussian lens (gas). Below the lens, at a depth of $1.5 \mathrm{~km}$, we placed a reflector that is partly horizontal (a reservoir) and partly dipping to the left. One can view the dipping part of the reflector as a model fault plane. Above the reflector, the Gaussian lens is embedded in a constant gradient $\left(0.45 \mathrm{~s}^{-1}\right)$ background; below the reflector the velocity is constant. This model is strongly refracting.

We synthesized multi-component elastic-wave data using a bandpass filter with dominant frequency $35 \mathrm{~Hz}$ as (isotropic, explosive) source wavelet, and a finite difference scheme. We extract the vertical component to suppress the shear-wave contributions. A typical shot gather over the lens (Figure 4, vertical component, shot position indicated by a vertical arrow in Figure 3 (left)) shows a complex pattern of reflections from the reflector propagated through the lens; we note the weak, remaining contributions from mode converted waves at later times that will not be treated properly by our acoustic-wave migration scheme here.

We migrated the data with the above mentioned depthextrapolation approach. An angle image gather is shown in Figure 5 (right). For comparison we show the angle image gather at the same location (left) reproduced from Brandsberg-Dahl et al. (2003), obtained by generalized 


\section{Kinematics of prestack shot-geophone migration}

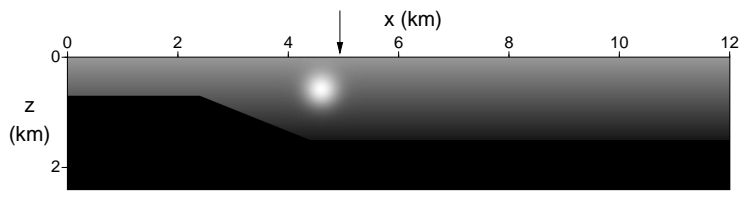

Fig. 3: Valhall lens model.

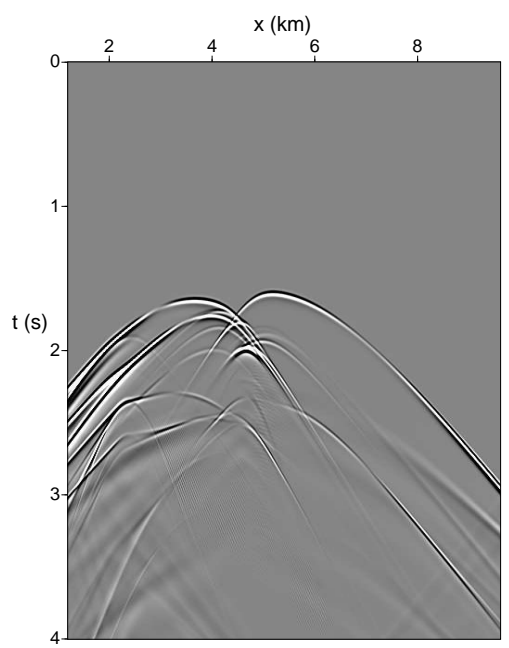

Fig. 4: Valhall lens model, shot record at shot location 4884 $\mathrm{m}$

Radon transform migration (without focussing in dip or the application of isochron filters). The left image gather is clearly contaminated by energetic non-flat events, while the right image gather is not.

\section{Conclusion}

The examples support the conclusions that were stated in the introduction and derived with ray-theoretical arguments above. Other examples, not shown here, exhibit the same behavior.

\section{References}

Brandsberg-Dahl, S., De Hoop, M. V., and Ursin, B., 2003, Focusing in dip and AVA compensation on scattering angle/azimuth common image gathers: Geophysics, 68, 232254 .

Claerbout, J., 1985, Imaging the earth's interior: Blackwell Scientific Publishers, Oxford.

De Bruin, C. G. M., Wapenaar, C. P. A., and Berkhout, A. J., 1990, Angle-dependent reflectivity by means of prestack migration: Geophysics, 55, 1223-1234.

De Hoop, M. V., Le Rousseau, J. H., and Biondi, B., 2003 , Symplectic structure of wave-equation imaging: A pathintegral approach based on the double-square-root equation: Geoph. J. Int., 153, 52-74.
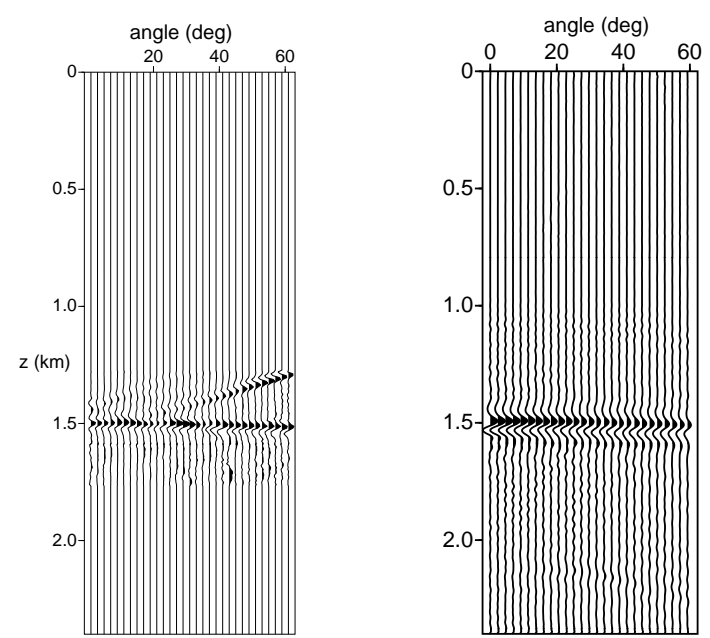

Fig. 5: Valhall lens model, common image point gathers obtained with the Kirchhoff angle transform (left) and the waveequation angle transform (right) at $4680 \mathrm{~m}$.

Le Rousseau, J. H., and De Hoop, M. V., 2001, Modeling and imaging with the scalar generalized-screen algorithms in isotropic media: Geophysics, 66, 1551-1568.

Nolan, C. J., and Symes, W. W., 1996, Imaging and conherency in complex structure: SEG, Expanded Abstracts, Society of Exploration Geophysicists, 66th Annual International Meeting, 359-363.

Nolan, C. J., and Symes, W. W., 1997, Global solution of a linearized inverse problem for the wave equation: Comm. P. D. E., 22, 919-952.

Prucha, M., Biondi, B., and Symes, W. W., 1999, Angledomain common image gathers by wave-equation migration: SEG, Expanded Abstracts,Society of Exploration Geophysicists, 69th Annual International Meeting, 824-827.

Sava, P., and Fomel, S., 2003, Angle-domain common-image gathers by wavefield continuation methods: Geophysics, 68, 1065-1074.

Schultz, P., and Sherwood, J., 1982, Depth migration before stack: Geophysics, 45, 376-393.

Stolk, C. C., and De Hoop, M. V., December 2001, Seismic inverse scattering in the 'wave-equation' approach, Preprint 2001-047, The Mathematical Sciences Research Institute, http://msri.org/publications/preprints/2001.html.

Stolk, C. C., De Hoop, M. V., and Symes, W. W., 2005, Kinematics of shot-geophone migration, Technical Report 05-04, Department of Computational and Applied Mathematics, Rice University, http://www.caam.rice.edu.

Stolk, C. C., and Symes, W. W., 2004, Kinematic artifacts in prestack depth migration: Geophysics, 69, 562-575.

Xu, S., Chauris, H., Lambaré, G., and Noble, M., 2001, Common angle migration: A strategy for imaging complex media: Geophysics, 66, no. 6, 1877-1894.

Yilmaz, O., 1987, Seismic data processing: Investigations in Geophysics No. 2. 